\title{
Monocular deprivation and the input to the visual cortex
}

\author{
from A. M. Sillito
}

THE role of visual experience in establishing the functional properties of the developing mammalian visual system is a topic which has aroused considerable controversy and has been the subject of a recent review in Nature (Barlow, Nature, 258, 199; 1975). There is however general agreement in this field that a normal input from the two eyes in early life is essential for maintaining an effective binocular input to visual cortical neurones. If monocular deprivation is produced in a kitten by suturing together the eye-lids of one eye at eye opening time for a period of two months or more it will be found that the majority of cortical neurones will be driven only by the non-deprived eye instead of both eyes as in a normal animal (Wiesel and Hubel, J. Neurophysiol., 26, 1003; 1963). There is a critical period during which monocular deprivation is effective in producing these changes. In kittens it extends from some time in the third week of life to approximately three months and the effects of deprivation extending through the critical period seem to be irreversible despite subsequent long periods of normal binocular vision (Hubel and Wiesel, $J$. Physiol., 206, 419; 1970). Even quite brief periods of monocular deprivation during the first month of the critical period will result in the deprived eye's appearing to lose access to cortical cells and an essentially complete takeover by the non-deprived eye (Peck and Blakemore, Expl Brain Res., 22, 57; 1975). However, reverse suturing (opening deprived eye, suturing the normal) within the critical period can result in the previously deprived eye's regaining its ability to drive cortical cells and a complete reversal of the original situation can even occur in which all the cells are driven by the previously deprived eye (Blakemore and Van Sluyters, J. Physiol., 237, 195; 1974).

Of particular interest at present are the mechanisms contributing to the effects of monocular deprivation. Although there are morphological changes in the layers of the lateral geniculate body (the thalamic relay between the eye and visual cortex) receiving an input from the deprived eye the functional properties of these cells seem to be relatively unchanged (Wiesel and Hubel, J. Neurophysiol., 26, 987; 1963). In fact binocular deprivation produces similar morphological changes in the lateral geniculate body but does not result in a dramatic loss of binocularly-driven neurones (Hubel and Wiesel, J. Neurophysiol., 28, 1029;
1965). This type of evidence together with the results of reverse suturing experiments carried out within the critical period have led to the view that there is a competitive interaction at the cortical level between the afferent terminals deriving from the respective eye inputs. It has been suggested that there may be an actual growth and reorganisation of afferent terminals (Movshon and Blakemore, Nature, 251, 504; 1974) with the implication of a loss on cortical cells of some or all the terminals deriving from the deprived eye. Alternatively, it can be argued that during the critical period synapses that are used become 'more effective', whilst those that are not are in some way 'silenced' although still present, and that within the critical period this is a reversible process. A recent paper (Duffy, Snodgrass, Burchfiel and Conway, Nature, 260, 256; 1976) has presented some important new evidence on this topic. They found, in cats monocularly deprived by lid suture from the fourth week of life to eight months, that intravenous injection of bicuculline revealed an input from the deprived eye to cells that were otherwise only driven by the normal eye. Thus they managed to reverse briefly the effects of monocular deprivation and furthermore they found that within this period, the receptive field properties in the two eyes were basically the same. The alkaloid bicuculline is an antagonist of $\gamma$-aminobutyric acid (GABA) in the central nervous system and GABA is a putative inhibitory transmitter in the retina, lateral geniculate body and visual cortex. Bicuculline applied iontophoretically to visual cortical neurones will produce changes in their receptive field properties that are consistent with a loss of an intracortical inhibitory input (Sillito, J. Physiol., $250,305 ; 1975)$. Hence the results of Duffy et al. suggest that the deprived eye actually retains an input to cortical cells but that this is in some way suppressed by GABA-mediated inhibitory process. It is tempting to view this simply as a block of intracortical inhibition but the presence of GABA-mediated inhibition at other levels in the visual system means that intravenously injected bicuculline will produce changes in excitability throughout the system and this may contribute to the observed effect.

Whilst no one would dispute that monocular deprivation from the fourth week of life to the eighth month produces an irreversible loss of the ability to drive cortical cells from the deprived eye, it must be noted that in the experi- ments of Duffy et al. the deprivation started in the fourth week and not at eye-opening time. This means that the animals had approximately one week of binocular visual experience partially overlapping the beginning of the critical period. This is reflected by the similarity of the receptive fields in the deprived eye to those in the normal, an occurrence which if deprivation had been from eye-opening time might not be expected in so far as the receptive fields would be more likely to be of the immature type (Blakemore and Van Sluyters, J. Physiol., 237, 195; 1975). It is important that the effects of intravenously injected bicuculline should be examined in a situation where monocular deprivation is started at eye-opening time, and hence where there will have been no opportunity for initial reinforcement of binocular input to cortical cells. Probably the most crucial experiment would be to examine the effects of iontophoretically applied bicuculline on visual cortical cells in monocularly deprived cats to see whether a local block of GABAmediated intracortical inhibition can reveal an input from the deprived eye. With the present evidence in mind there seems little doubt that some type of inhibitory suppression of the input of the deprived eye, either with or without a concurrent loss of synapses or synaptic effectiveness, constitutes an important component of the cortical effect of monocular deprivation.

\section{T antigen and DNA synthesis}

from Walter F. Mangel

Polyoma and SV40 virus induce an intranuclear tumour (T) antigen early in lytic infection in transformed cells and in tumour-bearing animals. $T$ antigen appears in lytically infected cells at the same time as the early virusspecific 19S mRNA and is followed by the induction of chromatin replication. This temporal sequence of events led to the hypothesis that $\mathrm{T}$ antigen is an early virus-coded protein which is responsible for the induction of cellular DNA synthesis that occurs after infection by papova viruses. In three recent articles from the laboratory of A. Graessmann in Berlin, direct experimental support consistent with this hypothesis is presented.

If $T$ antigen is the early gene product 